\section{(A) Check for updates}

Cite this: Polym. Chem., 2021, 12, 2035

Received 30th September 2020, Accepted 8th February 2021

DOI: $10.1039 /$ d0py01392g

rsc.li/polymers

\title{
Polymers and boron neutron capture therapy (BNCT): a potent combination
}

\begin{abstract}
Anaïs Pitto-Barry (D) *
Boron neutron capture therapy (BNCT) has a long history of unfulfilled promises for the treatment of aggressive cancers. In the last two decades, chemists, physicists, and clinical scientists have been coordinating their efforts to overcome practical and scientific challenges needed to unlock its full therapeutic potential. From a chemistry point of view, the two current small-molecule drugs used in the clinic were developed in the 1950s, however, they both lack some of the essential requirements for making BNCT a successful therapeutic modality. Novel strategies are currently used to design new drugs, more selective towards cancer cells and tumours, as well as able to deliver high boron contents to the target. In this context, macromolecules, including polymers, are promising tools to make BNCT an effective, accepted, and front-line therapy against cancer. In this review, we will provide a brief overview of BNCT, and its potential and challenges, and we will discuss the most promising strategies that have been developed so far.
\end{abstract}

\section{Introduction}

Boron neutron capture therapy (BNCT) is a two-component, or binary, radiotherapeutic modality. BNCT was initially developed in the clinic in the 1950 s by the neurosurgeon W. H. Sweet, while its therapeutic potential was discovered by

School of Chemistry and Biosciences, University of Bradford, Richmond Road, Bradford BD7 1DP, UK. E-mail: a.pitto-barry@bradford.ac.uk

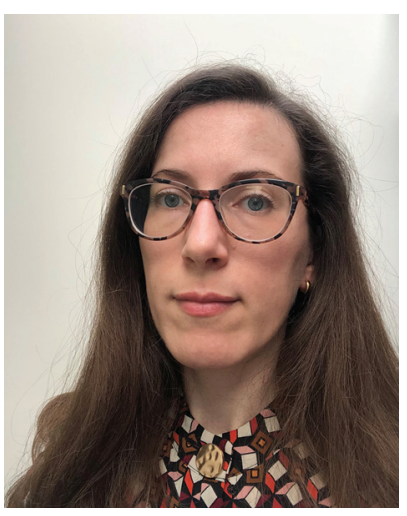

Anaïs Pitto-Barry
Anaïs became a Lecturer in Inorganic Chemistry at the University of Bradford in 2019. Her research interests include using polymers for the transport of inorganic compounds for biomedical applications. Previously, she obtained an MSc degree from ENSCR (France) and a PhD degree from the University of Neuchâtel (Switzerland) with Prof. Robert Deschenaux. In 2012, she initially joined the University of Warwick as a Swiss National Science Foundation Fellow to work with Profs Rachel O'Reilly and Andrew Dove. She also worked with Prof. Nicolas Barry on the applications of metallated compounds at the University of Warwick and the University of Bradford.
Locher in $1936 .{ }^{1}$ Some nuclides have a high propensity for absorbing low energy or thermal neutrons, and this is referred to as the neutron capture cross-section. ${ }^{10} \mathrm{~B}$ is one of the most attractive nuclides for neutron capture with a cross section of 3836 barns. It is a stable, non-radioactive isotope, with a natural occurrence of $20 \%$. The capture of thermal neutrons by ${ }^{10} \mathrm{~B}$ leads to the production of high linear energy transfer (LET) particles, alpha particles $\left(\mathrm{He}^{4}\right)$ and recoiling lithium-7 nuclei (Fig. 1). ${ }^{2}$ The path lengths of these particles are limited and approximately one cell in diameter $(10-14 \mu \mathrm{m})$, and there-

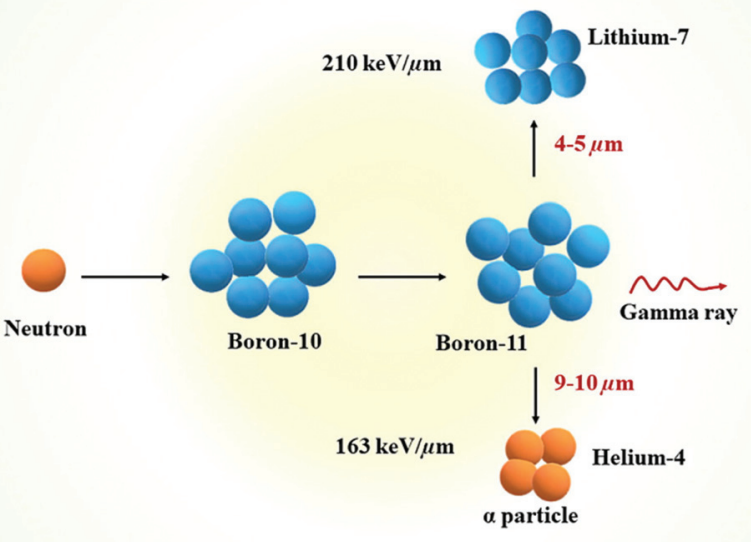

Fig. 1 Schematic representation of the nuclear reaction that happens during the BNCT process. Reproduced from ref. 2 with permission from Elsevier, copyright 2020. 
fore only cells that have internalised ${ }^{10} \mathrm{~B}$ are impacted by irradiation. Indeed, the neutron capture cross section of hydrogen and nitrogen is weak enough to prevent any damage to biological tissues from their reaction with thermal neutrons (0.3326 and 1.91 barns, respectively). This nuclear reaction leads to the destruction of a wide variety of biologically active molecules (e.g., DNA, RNA, and proteins) whilst the surrounding tissues remain unaffected.

For successful BNCT treatment, a critical amount of ${ }^{10} \mathrm{~B}$ and a sufficient number of thermal neutrons are therefore needed to be delivered to individual tumour cells.

Ideal boron compounds should exhibit the following characteristics:

- low systemic toxicity;

- low healthy tissue uptake and high tumour uptake;

- high tumour/blood and tumour/surrounding normal tissue concentration ratios (greater than 3-4:1). This minimises the impact that the capture of neutrons by hydrogen and nitrogen, the two elements present in high concentrations in the body, can have on the absorbed radiation dose;

- tumour concentrations of $20-35 \mu \mathrm{g}$ of ${ }^{10} \mathrm{~B}$ per gram of tumour, which accounts for $10^{9}$ atoms of ${ }^{10} \mathrm{~B}$ per cell;

- rapid clearance from blood and normal tissues;

- persistence in the tumour for the duration of the treatment. ${ }^{3}$

This review will first introduce the early stages of BNCT in terms of the compounds used, before focusing on high-molecular weight polymeric systems that have been used for in vitro and in vivo studies for potential BNCT applications.

\section{First generation of compounds}

The first boron-containing molecules used for BNCT were not designed for such an application, but were selected because of their availability, known pharmacology, and lack of toxicity. Boric acid $\left(\mathrm{H}_{3} \mathrm{BO}_{3}\right)$, sodium tetraborate (borax) and their derivatives were initially used. The first experiments of neutron irradiation were conducted on samples of mammary carcinoma, lymphoma and undifferentiated sarcoma grown on mice and infused with boronic acid. The tissues were shown not to regenerate in vivo. These experiments carried out in the 1930-40s were the first demonstration that neoplastic cells could be destroyed in vivo with BNCT treatment. ${ }^{4}$

Early clinical trials of BNCT were conducted in the US at the Brookhaven National Laboratory Reactor using thermal neutrons and sodium tetraborate during the 1950s on malignant gliomas that were resisting all forms of treatment. ${ }^{5}$ Between severe damage of the scalp and no significant prolongation of survival nor evidence of therapeutic efficacy, clinical trials in the US were stopped in $1961 .^{6}$

\section{Second generation of compounds}

Following the poor clinical outcomes of the derivatives of boric acid, a second generation of compounds was investigated,

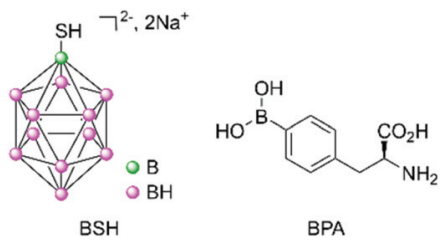

Fig. 2 Chemical structures of BSH, a boron cluster (left), and BPA (right).

initially in Japan for brain tumours from the late 1960s. ${ }^{7}$ Boron clusters with one or two sulfur atoms showed some success, with the presence of the mercapto moieties being hypothesised to allow binding to antibodies via a thiol-disulfide exchange. ${ }^{8}$ Of these boron clusters, sodium borocaptate (BSH, $\mathrm{Na}_{2} \mathrm{~B}_{12} \mathrm{H}_{11} \mathrm{SH}$, Fig. 2) is the most promising owing to its high and persistent boron concentrations in tumour and low systemic toxicity even if it exhibits a low mean tumour/ blood ratio. $p$-Boronophenylalanine (BPA, Fig. 2) is another compound which received a lot of interest, especially in the treatment of malignant melanoma. Malignant melanoma cells preferentially take up BPA because its chemical structure is similar to that of tyrosine, which is required for melanogenesis. Other boron-containing amino acids were synthesised following the promising clinical trial results obtained for BPA.

Clinical trials have been carried out since the 1960s with these second-generation compounds, either alone or in combination with another treatment regimen (surgery or radiotherapy). ${ }^{9}$ Completed phase I/II studies have shown encouraging results for difficult-to-treat and recurrent head and neck cancers, melanoma, and gliomas (by intratumoural exposure) in Japan, Taiwan, USA, and in several European countries (Finland, Germany, Austria, France, and UK). ${ }^{10,11}$ Owing to the progress of nuclear physics, it is now possible to produce lowenergy thermal neutrons using accelerators rather than nuclear reactors. ${ }^{12}$ This has allowed for easier access to such neutrons. For example, one recently reported phase II study using $\mathrm{BPA}-{ }^{10} \mathrm{~B}$ has used BNCT in a Japanese urban hospital setting on patients with unresectable carcinoma of the head and neck, with very promising results. ${ }^{13}$

Despite this encouraging progress made by physicists and clinicians, both BSH and BPA do not fulfil the criteria needed for efficient boron delivery agents (e.g., poor uptake, low selectivity between healthy and cancer cells). These compounds were selected because they were the best benchmark molecules available to gauge the progress made with the technology. With better facilities and technical understanding of the methodology, a third generation of compounds for making BNCT a viable therapeutic modality was therefore needed.

\section{Third generation of compounds}

Many boron delivery agents have been designed in the last few decades, ranging from low- to high-molecular weight com- 

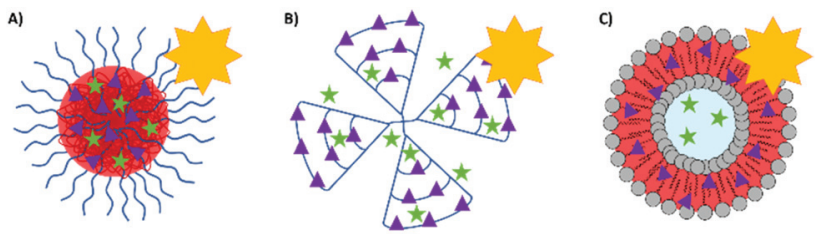

$\Delta$ Conjugated boron

Encapsulated boron

䇏 Targeting and/or labelling moieties

Fig. 3 Overview of the various polymeric systems containing boron, either conjugated or encapsulated, and the possible conjugation to targeting or labelling moieties: (A) micelles made from polymers or dendronised polymers; (B) dendrimers; and (C) liposomes.

pounds to overcome the limitations of BPA and $\mathrm{BSH}$, with a focus on selective tumour cell targeting and the delivery of therapeutic concentrations of boron. They can be classified into three different types of boron-carriers: boron-containing small molecules (which will not be discussed here), boron compound conjugates and boron delivery nanoparticles (Fig. 3). Many systems have also been designed to incorporate biomolecules. This bioconjugation of synthetic (macro)molecules with proteins has opened the way for the design and synthesis of new molecules with enhanced chemical properties and functions. Proteins, DNA, RNA or carbohydrates have been derivatised for applications in ligand discovery, disease diagnosis, and disease treatment. ${ }^{14}$

\section{Boron compound conjugates}

Polymeric systems. The chemistry of boron is well established to produce small molecules containing usually only one boron, with the main focus of such molecules being towards catalysis $^{15}$ or functional nanostructured materials. ${ }^{16,17}$ However, most polymeric systems developed with BNCT in mind use carborane derivatives as boron compounds, owing to their rich boron composition.

Carborane-conjugated polymers are of high interest for designing potential agents for BNCT. After their initial work on the direct incorporation of carborane moieties in welldefined macromolecules by living free-radical polymerisation and sequential dendrimer synthesis, ${ }^{18}$ Adronov and coworkers designed, in 2005, a carborane acrylate monomer that could be copolymerised by atom transfer radical polymerisation (ATRP) to obtain water-soluble polymers. Whilst no biological studies were conducted, the different synthesised materials were tested for their neutron irradiation potential and showed the release of gamma radiation, thus confirming their potential as neutron capture systems. ${ }^{19}$

In 2016, Huang and co-workers synthesised amphiphilic carborane-conjugated polycarbonates by ring-opening polymerisation of a carborane cyclic carbonate monomer onto a poly(ethylene glycol) macroinitiator (Scheme 1). ${ }^{20}$ The boron content was tuned by varying the ratio between the two blocks and the molecular weight of the initiator. Different sizes of polymeric micelles were obtained; the larger ones (diameter of $150 \mathrm{~nm}$ ) showed more capture by the liver despite the PEG chains on the shell, while the smaller ones (diameter of

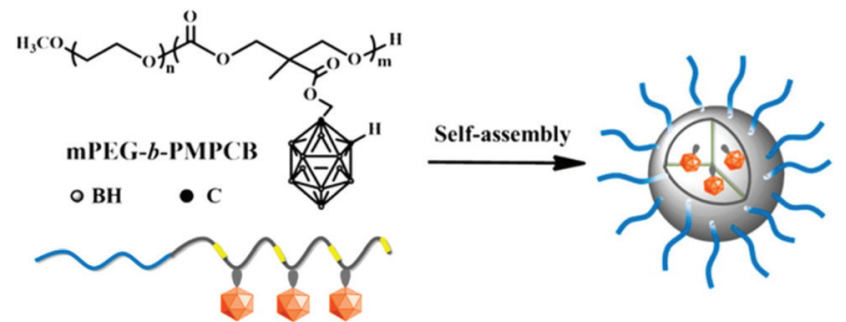

Scheme 1 Structure of the amphiphilic carborane-conjugated polycarbonate and self-assembly in water, adapted from ref. 20 with permission from the American Chemical Society, copyright 2016.

$50 \mathrm{~nm}$, with shorter PEG chains) were more likely to be internalised into cancer cells. No toxicity was observed for the range of nanoparticles on normal mouse fibroblast L929 cells and human cervical cancer HeLa cells, while the carboranecontaining monomer exhibited a 50\% inhibitory concentration of $1.8 \mathrm{mM}$ on L929 cells which is higher than other small molecules with carborane entities. ${ }^{21}$ Finally, BNCT experiments were performed on mice bearing U14 tumours (mouse cervical cancer cell line) after sufficient injection of the micelles was established. No reduction of tumour volume was observed without irradiation, while an irradiation time of one hour led to significant shrinking of the tumours. Importantly, mice did not lose weight for over 21 days after irradiation.

In 2016, the radical addition-fragmentation chain-transfer (RAFT) technique was used to polymerise a carborane-containing methacrylate monomer onto a poly(caprolactone) (PCL) macroinitiator. $^{22} \mathrm{~A}$ further block made of oligo(ethylene glycol)methyl methacrylate (OEGMA) was then added to obtain an amphiphilic triblock copolymer that was functionalised on the alcohol end group of the PCL block with a near-infrared aminocyanine dye (Fig. 4). Indeed, imaging-guided BNCT is crucial to determine the distribution of carborane-containing nanoparticles in the body and their accumulation within organs and organelles. The biocompatibility of the micelles obtained by solvent displacement was determined against hepatic cancer and normal cell lines and low cytotoxicity was observed in both cases (cell viability of more than $90 \%$ up to $\left.0.5 \mathrm{~g} \mathrm{~mL}^{-1}\right)$. In vitro fluorescence studies showed that the dye accumulated in the cytoplasm of cells, which hints at the suc-

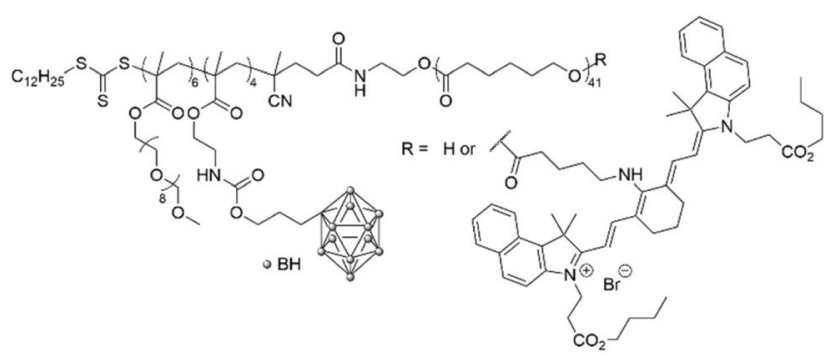

Fig. 4 Structure of the amphiphilic poly(carborane-containing methacrylate)- $b$-poly(caprolactone) with or without a near-infrared aminocyanine dye. 
cessful accumulation of the polymeric boron-containing micelles in the cytoplasm, even if no degradation studies to look at the stability of the micelles were conducted.

In 2014, Wu and co-workers used a slightly different approach to produce polymeric nanoparticles by mimicking the structure of a small surfactant with the carborane unit as the hydrophobic head covalently linked to a $\mathrm{PEG}_{2000}$ unit, the hydrophilic tail, via the thiol-ene reaction. ${ }^{23}$ Such polymeric chains were further labelled with the hydrophilic rhodamine $\mathrm{B}$ dye on a second available position on the carborane unit (Scheme 2). Self-assembly by solvent displacement led to vesicles with a diameter of around 160-170 $\mathrm{nm}$ and a shell thickness of $10 \mathrm{~nm}$, depending on the absence or presence of the dye. The vesicles did not exhibit cytotoxicity on HepG2 cells (liver cancer cells) and had a much longer in vivo circulation time (60\% after $24 \mathrm{~h}$ ) than $\mathrm{BSH}$ alone $(20 \%)$ or other carborane-containing PEG formulations. In vitro fluorescence studies on U87 cells (glioblastoma cancer cells) showed the accumulation of the dye in the cytoplasm. Using fluorescence microscopy after intravenous injection of a saline solution of the fluorescent vesicles, the vesicles were found to accumulate in the spleen of mice but not in other organs (heart, liver, kidney), which confirms their potential as drug delivery systems.

In 2019, Tsukahara and co-workers coated the surface of a vesicle with a copolymer containing a 2-methacryloyloxyethyl phosphorylcholine zwitterionic block and a methacrylamide benzoxaborole second block which, at $\mathrm{pH}$ above 7.6, forms ionised $\mathrm{B}(\mathrm{OH})_{2}{ }^{-}$groups capable of binding to monosaccharides such as galactose (Fig. 5). ${ }^{24}$ The vesicle used is the hemagglutinating virus of Japan Envelope (HVJ-E) which is a non-proliferative and non-infectious vesicle utilised as a "non-viral" transfection reagent based on its high cell-fusion affinity. Unusually, this system did not use the capability of vesicles to load materials within their internal core. ${ }^{25}$ The polymer was synthesised by free radical polymerisation, purified by dialysis and freeze-dried before being used for the coating of HVJ-E. The obtained bioconjugates were still of a spherical shape and dilution of the solution did not affect the diameter of the bioconjugates. $10^{7}-10^{8}$ atoms of boron were found to be on the surface of one particle, which means only a few bioconjugates need to bind to one cell to reach the needed $10^{9}$ boron atoms per cell for efficient boron neutron capture. The polymer generated no apparent cytotoxicity on HepG2 cells (human liver carcinoma cells). In vitro fluorescence studies of the bioconju-

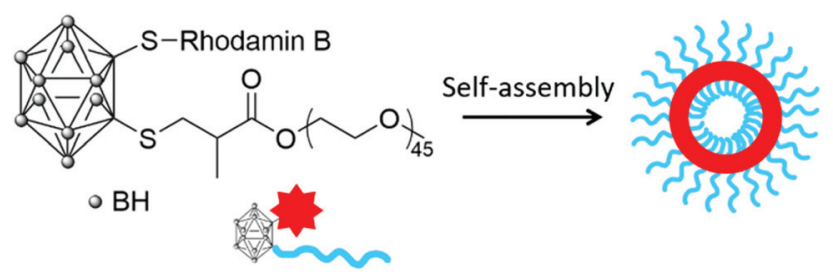

Scheme 2 Self-assembly into vesicles of a fluorescent carborane-conjugated POEGMA.

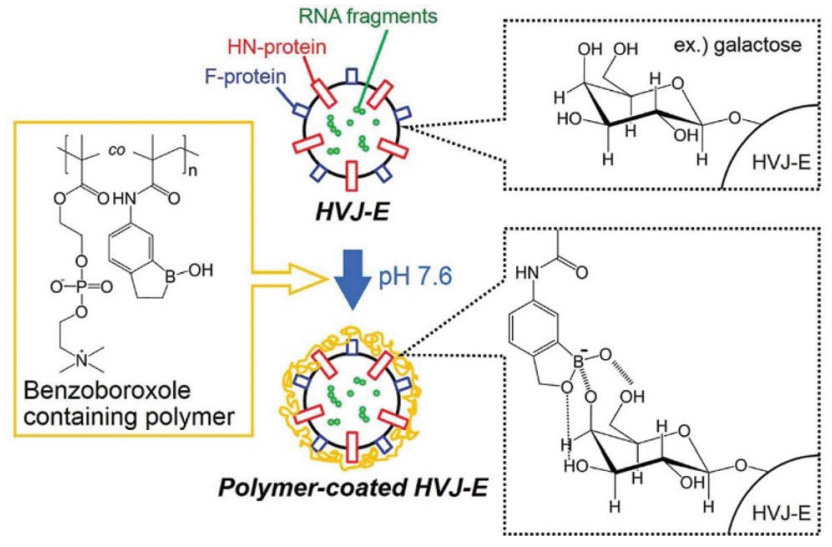

Fig. 5 Schematic illustration of HVJ-E surface modification by a benzoxaborole-containing polymer. Reproduced from ref. 24 with permission from Taylor \& Francis, copyright 2019.

gates revealed that the intracytoplasmic localisation of the polymer took between 45 and 90 minutes. No in vivo or BNCT experiments have been reported so far using this system.

Recently, amphiphilic diblock copolymer nanoparticles with a boron-rich core have been synthesised by RAFTmediated polymerisation-induced self-assembly (Fig. 6). ${ }^{26} \mathrm{~A}$ methacrylic monomer with a pinacol boronate ester was polymerised by RAFT onto various hydrophilic POEGMA blocks used as macromolecular transfer agents to produce sub-100 nm spherical polymeric micelles. Nanoparticles were shown to be non-cytotoxic up to a concentration of $100 \mu \mathrm{g}$ $\mathrm{mL}^{-1}$. The uptake of the nanoparticles was followed by adding a fluorescent tag. Hsu, Delaittre and co-workers compared their systems with BPA, in terms of solubility and boron content, and found that such nanoparticles are indeed more advantageous than the small molecule.

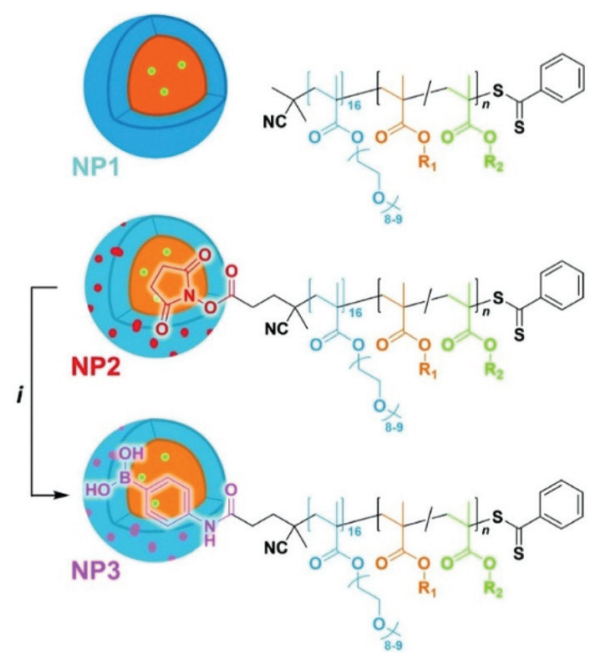

Fig. 6 Schematic illustration of the POEGM $-b$-poly(4-pinacolboronylbenzyl methacrylate). $R_{1}=4$-phenylboronate pinacol ester, $R_{2}=$ fluorescein. Reproduced from ref. 26 with permission from the Royal Society of Chemistry, copyright 2020. 
Peptides have also been used to incorporate boron atoms and deliver them to specific receptors. Barth, Soloway and coworkers functionalised poly(DL-lysine) with isocyanato-closododecaborate, which was further linked to a monoclonal antibody. ${ }^{27,28}$ This polymeric linker was used to minimise the reduction of the immunoreactivity of the monoclonal antibody in the presence of boron clusters. The bioconjugates contained more than 1000 boron atoms per macromolecule and retained between 40 and $90 \%$ of the immunoreactivity, thus making them potentially suitable as delivery systems for BNCT. This initial approach was, however, limited by polymers with a molar mass dispersity relatively important, which in turn led to heterogeneity in the number of boron atoms present.

In 2020, Beck-Sickinger and co-workers described the use of a peptide conjugate to selectively target the human $\mathrm{Y}_{1}\left(\mathrm{hY}_{1}\right)$ receptor, which is overexpressed on cancer cells. ${ }^{29}$ This 36-amino acid peptide was functionalised on two lysines with deoxygalactopyranosyl-modified carborane building blocks. These saccharide moieties ensured appropriate aqueous solubility and allowed the incorporation of up to 80 boron atoms per peptide. In vitro studies on MCF-7 cells (human breast cancer cells) showed that the bioconjugate systems exhibited high receptor activity and internalisation at the $\mathrm{hY}_{1}$ receptors, selectivity against other hY receptor subtypes and low intrinsic cytotoxicity.

Dendrimers. Dendrimers have received a lot of attention as drug-delivery systems, owing to their large number of periphery sites available for functionalisation and the well-advanced methodologies available to synthesise them. Functionalisation allows for the introduction of a variety of molecules, ranging from boron-containing synthons, to polymer chains and biomolecules such as antibodies.

Poly(amidoamine) (PAMAM) dendrimers have particularly been studied. ${ }^{30}$ Barth and co-workers have extensively studied boronated PAMAM dendrimers conjugated with various biomolecules such as monoclonal antibodies. ${ }^{31}$ Monoclonal antibodies are promising in the treatment of cancers. By recognising specific proteins on the membrane of cancer cells, monoclonal antibodies can selectively target cancer cells. The epidermal growth factor receptor (EGFR) gene is often amplified in human glioblastomas, with tumour cells having up to 100 times more receptors than normal cells. Such an EGPR can therefore be used as a target, using EGF bioconjugates to deliver boron atoms to cancer cells. Barth and coworkers developed several methodologies to boronate EGF, ${ }^{32}$ such as using the thiol-maleimide "click" chemistry reaction. Starburst dendrimers composed of repetitive polyamidoamino (PAMAM) groups were functionalised on their terminal amine groups by an isocyanato polyhedral borane and further functionalised with a thiol group to react with the maleimide groups of EGF derivatives (Fig. 7). The stable bioconjugates bearing a fourthgeneration dendrimer were shown to contain 960 atoms of boron per molecule. ${ }^{33}$ By taking into account the density of receptor sites on the cancer cells, and the affinity constant, the authors determined that each molecule of the antibody must deliver 1000 atoms of boron to attain a critical local boron con-

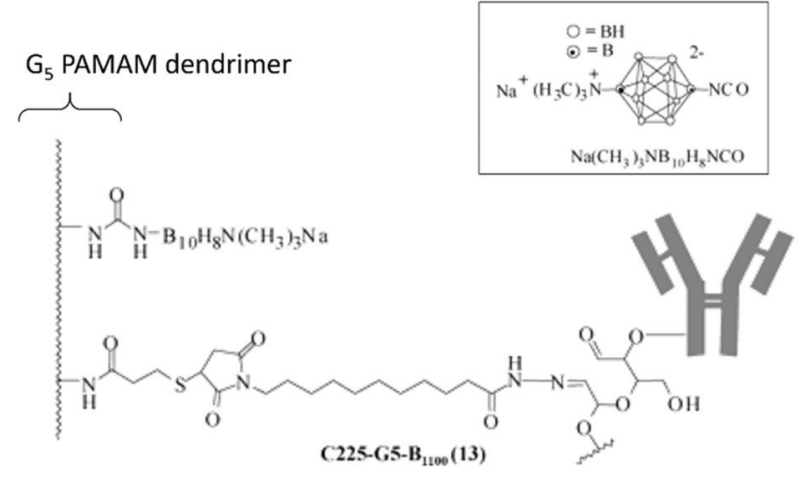

Fig. 7 Boron-containing PAMAM dendrimer linked to an antibody. Adapted from ref. 31 with permission from the American Chemical Society, copyright 2004.

centration. The boron concentrations in $\mathrm{F} 98_{\mathrm{WT}}$ and F98 $8_{\mathrm{EGFR}}$ cells (cancer brain rat cells) implanted in rats were found to be 9.2 and $21.1 \mu \mathrm{g} \mathrm{g}^{-1}$ of tumour 24 hours after injection, while no boron could be detected in the liver, spleen, kidneys, brain and blood. The mean survival time (MST) in the F98 ${ }_{\text {EGFR }}$ rat model after BNCT treatment was longer with the bioconjugate compared to BPA (45 versus 39 days). ${ }^{34}$

The initial focus of BNCT was the treatment of glioblastomas, which could not be treated by other therapeutic modalities. Therefore, the ability to cross the blood-brain barrier (BBB) needed to be investigated. In 2002, Barth and co-workers examined the use of convection-enhanced delivery (CED) to improve the tumour uptake of a heavily boronated dendrimer EGF bioconjugate. ${ }^{35}$ Indeed, the high molecular weight of the EGF moiety is a limiting factor for accessing the brain. Convection-enhanced delivery is a delivery method that can bypass the BBB and introduce agents directly into the extravascular space of the central nervous system. Results on F98 $8_{\text {EGFR }}$ glioma-bearing rats showed that the delivery of these high-molecular-weight boronated dendrimer EGF bioconjugates was improved compared to intravenous injection with an increased volume of distribution within the tumour.

The antibody IB16-6 was also conjugated by Barth, Soloway and co-workers to PAMAM dendrimers, which were functionalised with a decaborane cluster. ${ }^{36}$ The antibody was linked to the outer shell of the dendrimer by a derivatised site, which prevents a random attachment and retains its localising properties in in vitro studies. However, in vivo studies on rats bearing B16 melanomas showed a tendency to accumulate in the liver and spleen and a lack of specificity for the tumour.

To further enhance the efficacy of BNCT, the vascular endothelial growth factor (VEGF) receptors, which are overexpressed in tumour neovasculature, can also be targeted. ${ }^{37}$ Barth and co-workers used the coupling strategy reported for EGFR bioconjugates to bind four boronated PAMAM dendrimers to one VEGF molecule (Fig. 8). ${ }^{38}$ The activity of the protein was not affected by a large increase in molecular weight. By labelling these bioconjugates with the near-IR Cy5 dye, and following internalisation via receptor-mediated endocytosis, boron could 


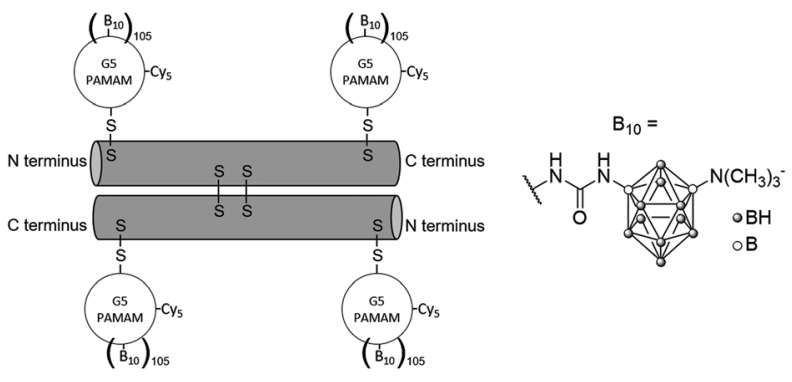

Fig. 8 VEGF-containing bioconjugate made of $G_{5}$ PAMAM dendrimers functionalised by the near-IR dye Cy5 and the boron cluster dodecaborate (a total of 105-110 boron clusters per dendrimer).

be imaged in the perinuclear region where angiogenesis is the most active in the 4T1 breast carcinoma mouse tumour model.

The biodistribution of dendritic prodrugs can be improved by adding poly(ethylene glycol) (PEG) chains to the surface of dendrimers. Indeed, by coating the surface of drug-delivery systems with flexible and hydrophilic polymers (PEG and polyoxazoline), longer circulation times (i.e., stealth properties) have been observed, which can then lead to a higher probability to access tumour sites and their leaky vasculature. ${ }^{39}$ In 2003, Tjarks and co-workers functionalised a series of boronated G3 PAMAM dendrimers bearing PEG chains and folic acid (FA), to minimise hepatic uptake and increase receptor targeting, respectively. ${ }^{40}$ The best results in terms of tumour selectivity were obtained with a G3 boronated dendrimer coated with only one $\mathrm{PEG}_{2000}$, while a few shorter chains $\left(\mathrm{PEG}_{500}\right.$ or $\left.\mathrm{PEG}_{750}\right)$ did not effectively shield the dendrimer surface and a large number of short PEG chains did not shield the dendrimer from opsonisation. However, both hepatic and renal uptakes were still very high.

In 2012, Hosmane and co-workers synthesised phenylenecored small dendrimers containing three, six or nine peripheral $o$-carborane clusters (Fig. 9). ${ }^{41}$ The in vitro biological evaluation of the dendrimer with nine carborane units showed that it accumulated either in human hepatocellular carcinoma cells or bound to their surface, with a boron concentration of up to $2540 \mathrm{ng}$ per $5 \times 10^{5}$ cells after 20 hours of incubation.

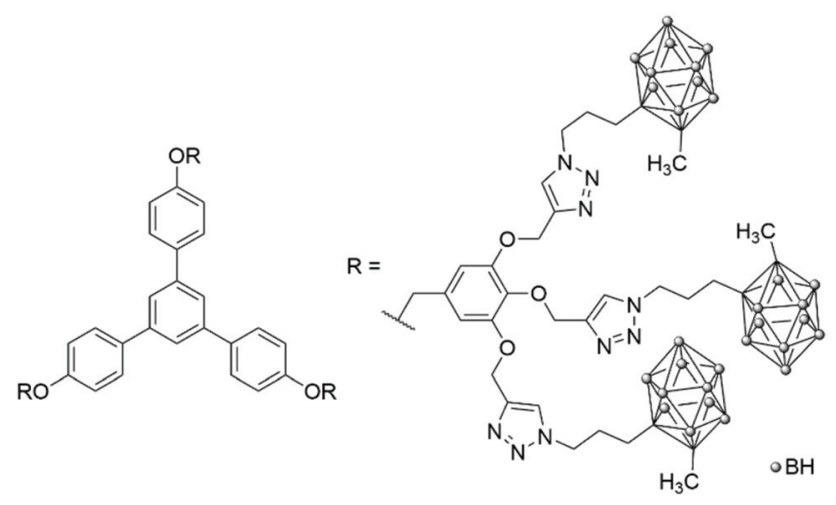

Fig. 9 Phenylene-cored carborane dendrimers prepared by click chemistry.
Such a concentration corresponds to about $1.5 \mathrm{mg}$ of boron per gram of tumour, which, by taking into account the natural abundance of ${ }^{10} \mathrm{~B}$, still gives a value of $300 \mu \mathrm{g} \mathrm{g}{ }^{-1}$, above the $30 \mu \mathrm{g}$ of ${ }^{10} \mathrm{~B}$ per $\mathrm{g}$ of tumour required for efficient BNCT. No further studies have been carried out on such systems. Hosmane and co-workers also explored the use of a different core, made of gold nanoparticles, which is then functionalised by dendrons with thiol moieties on the apex and with carborane and PEG units at the periphery. ${ }^{42}$ These water-soluble gold nanoparticles have, however, not been tested as BNCT materials.

The bifunctional carborane synthon strategy developed by Adronov and co-workers for dendritic polymers was also used to incorporate carborane units into an aliphatic polyester dendrimer (Fig. 10). ${ }^{43}$ Such localisation of the carborane allows for the presence of peripheral hydrophilic groups, which improves the water solubility of the dendrimer and masks the hydrophobic carborane unit. The 2,2-bis(hydroxymethyl)propanoic acid (bis-MPA) core of the dendrimers, which is biocompatible, non-immunogenic, non-toxic, water soluble, and tolerated in vivo, was functionalised by a carborane unit. The second functional and opposite site of this carborane unit was further used to incorporate more bis-MPA units and therefore to increase the overall size of the dendrimer and its aqueous solubility with a value of $8 \mathrm{mg} \mathrm{mL} \mathrm{mL}^{-1}$ observed for the dendrimer containing four carborane units and 64 peripheral hydroxyl groups. Neutron activation experiments on the various dendrimers fully solubilised in THF revealed that they all exhibit the characteristic gamma radiation signature at $480 \mathrm{keV}$, which confirms that boron neutron capture events take place. Analysis of the samples after six hours of irradiation showed no degradation, which is expected given only $0.01 \%$ of the ${ }^{10} \mathrm{~B}$ atoms reacted within the time frame of the experiment.

Dendritic polymers. Dendritic polymers are highly branched polymers with three-dimensional architectures. There are

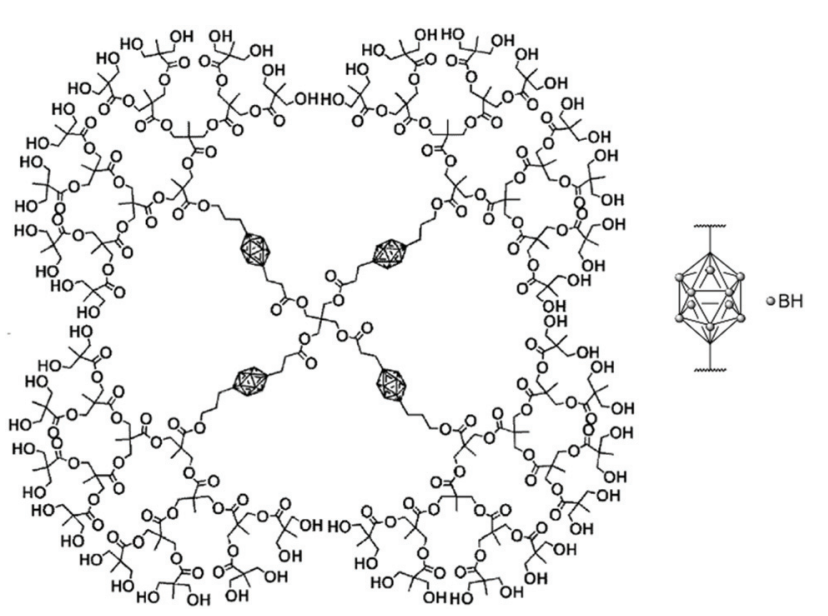

Fig. 10 Structure of the fourth-generation bis-MPA dendrimer bearing four bifunctional carborane units and 64 hydroxyl groups. Adapted from ref. 43 with permission from the American Chemical Society, copyright 2005. 
many sub-classes: dendrimers (as discussed above), hyperbranched polymers, multi-arm star polymers, dendronized polymers, hypergrafted polymers and dendritic-linear block copolymers. Dendritic polymers exhibit unique chemical and physical properties derived from their architecture: they possess a large number of terminal functional groups, low viscosity and good solubility. The fine-tuning of their size, composition, degree of branching, and further functionalisation with targeting ligands or imaging agents makes them suitable candidates for biomedical applications. ${ }^{44}$

Hawthorne and co-workers developed closomer species, which are dendrimer-like structures. ${ }^{45}$ The core of the structure was made of the rigid icosahedral boron scaffold [closo$\left.\mathrm{B}_{12}(\mathrm{OH})_{12}\right]^{2-}$ anion while the shell consisted of twelve $o$-carborane units linked through ester and ether functions to the central boron core. Aqueous solubility was improved by modifying the pendant carborane units. These unimolecular nanoparticles (with a diameter of around $3 \mathrm{~nm}$ ) were well tolerated by mice and are not toxic. Biodistribution data were collected on mice bearing EMT6 tumours (breast cancer cells) and showed that the maximum tumour boron concentration was achieved 24 hours post-injection and reached $10.5 \mu \mathrm{g} \mathrm{B} \mathrm{g}{ }^{-1}$ of tumour, with a tumour-to-blood ratio of 9.4.

In 2008, Adronov and co-workers extended their library of synthons containing carborane units by functionalising one side of the carborane with a styrene entity and the other side with the aliphatic polyester dendrimer backbone, which is water soluble, biocompatible and biodegradable. ${ }^{46}$ After nitroxide-mediated polymerisation and divergent growth of the dendrons, high-molecular-weight polymers with narrow dispersity were obtained, with a theoretical boron content of around $12 \%$ and a water solubility of around $1 \mathrm{mg} \mathrm{mL}^{-1}$ (Fig. 11).

\section{Boron-loaded systems}

Dendrimers. Dendrimers, owing to their unique 3D structure, contain cavities between branches. These cavities can be used to encapsulate different materials, both organic and inorganic, and to perform various reactions. ${ }^{47}$

A monoclonal antibody directed against the stem cell marker CD133, frequently expressed in the membrane of glioma stem cells, was used by Sun et al. to deliver a heavily boronated poly(amido amine) dendrimer. ${ }^{48} \mathrm{BSH}$ was loaded

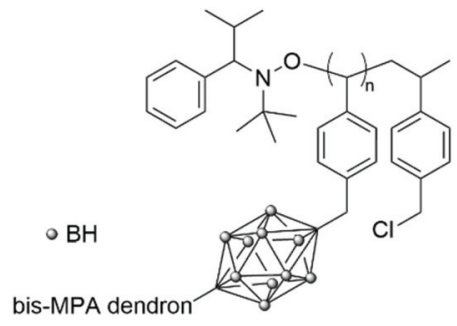

Fig. 11 Styrenic dendritic polymer bearing carborane units functionalised with a bis-MPA dendron.

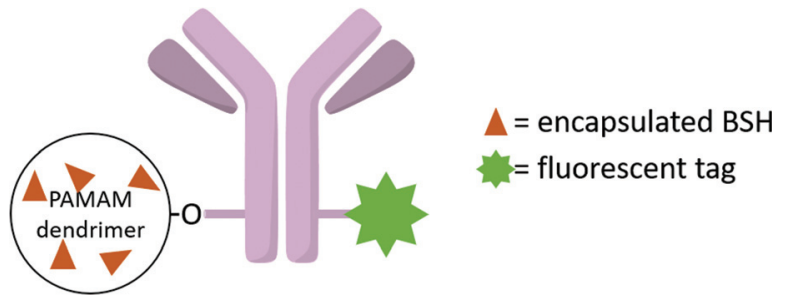

Fig. 12 Monoclonal antibody targeting nanoparticle containing BSH encapsulated within the PAMAM dendrimer.

into the voids available within the structure of the PAMAM dendrimer (Fig. 12). Glioma stem cells are resistant cells that escape conventional radiotherapy- and chemotherapy-induced cell death and lead to tumour regrowth. In vitro studies on CD133+ and CD133- SU2 (glioma stem) cells confirmed that more than $10^{9}$ boron atoms were delivered to the CD133+ SU2 cells, with some selectivity compared to the CD133- SU2 cells, therefore reaching the minimum amount of boron per cell established as needed for BNCT. In vivo studies on $\mathrm{BALB} / \mathrm{c}$ mice bearing intracerebral CD133+ SU2 cells confirmed the boron uptake and these selectivity and biodistribution studies showed the influence of the nanocarrier on the boron ratio between the tumour and the brain or the blood. BNCT treatment led to a significantly longer survival time in BALB/c mice bearing intracerebral CD133+ SU2 cells compared to that of CD133- SU2 cells. The MST was even longer when both the boron-containing bioconjugate and free $\mathrm{BSH}$ were administered before neutron irradiation.

Virus. HVJ-E loading capability has been used by Lee and coworkers to deliver BSH for the treatment of liver tumours. ${ }^{49}$ $\mathrm{BSH}$ was incorporated into an inactive form of the virus, which is used as a carrier agent following a standard procedure that allows the incorporation of small molecules inside the virus envelope. Then, a natural polymer, cationised gelatin, was mixed with HVJ-E to reduce its hemagglutinating activity, which is a limiting factor for the in vivo circulation of HVJ-E in the bloodstream. This allowed its use for systemic administration. In mice bearing multiple LM8G5 liver tumours, the use of this carrier was shown to increase the concentration of boron in the cytoplasm and the nucleus of tumour cells and the tumour/normal liver ratio compared to free $\mathrm{BSH}$. BNCT experiments in vivo with the boron-loaded bioconjugate exhibited a significant increase of the mean survival time of tumour-bearing mice compared to $\mathrm{BSH}$ at a comparable dosage of ${ }^{10} \mathrm{~B}$.

Liposomes. Liposomes have extensively been used in therapeutic and diagnostic applications owing to their unique properties as they can entrap hydrophilic drugs and prevent their premature release in the bloodstream. Liposomes became the first nanomedicines in FDA clinical trials with the approval in 1995 for Doxil®, a PEGylated liposome formulation that encapsulates doxorubicin. ${ }^{50}$

The first example of a ${ }^{10} \mathrm{BSH}$-encapsulated liposome conjugated with a monoclonal antibody was reported in 1989 by 
Yanagië and co-workers. Up to $1.3 \times 10^{4}{ }^{10} \mathrm{~B}$ atoms were encapsulated per antibody-conjugated liposome. ${ }^{51}$ The immunoliposomes were shown to in vitro selectively reach tumour cells and to suppress growth after thermal neutron irradiation.

In 1992, Hawthorne and co-workers encapsulated different borane anions inside phospholipid liposomes using their water-soluble sodium salts. ${ }^{52}$ In vivo studies in tumour-bearing mice revealed that good affinity of the liposomes for tumour cells was observed despite the absence of targeting entities. Boron concentrations in the tumour tissue were found to reach the therapeutic range ( $15 \mu \mathrm{g}$ of boron per $\mathrm{g}$ of tumour) for boron clusters containing 20 atoms of $\mathrm{B}$.

Various boron-encapsulated liposomes have been developed during the last few decades, however, most formulations did not contain enough boron within their core, which prevents them from reaching the therapeutic range needed for BNCT. ${ }^{53}$

In 2019, Krishnan, Prasad and co-workers designed an azolectin phospholipid-based liposome system to encapsulate both small boron nanoparticles and the near-infrared fluorescent dye Cy5 (Fig. 13). ${ }^{54}$ The boron nanoparticles (hydrodynamic diameter of $15 \mathrm{~nm}$ ) were prepared using $\mathrm{CO}_{2}$ laserinduced pyrolysis mixtures of $\mathrm{B}_{2} \mathrm{H}_{6}$ and $\mathrm{SF}_{6}$ and loaded inside the liposomes by the water-in-oil emulsion method. The targeting moiety FA was conjugated on the surface of the liposomes by EDC/NHS coupling, which increased the hydrodynamic diameter of the liposomes from 100 to $120 \mathrm{~nm}$. Cellular uptake studies were performed in rat C6 glioblastoma cells, with an enhanced uptake for the FA-conjugated liposomes. The ability of the liposomes to cross the BBB was determined with a $2 \mathrm{D}$ in vitro BBB model made of human brain microvascular endothelial cells and normal human astrocytes. While both nonand FA-conjugated liposomes crossed the BBB without damage to their structure, the crossing was more effective for the FA-conjugated liposomes, as illustrated by the higher boron content in cells determined by ICP-MS $3.7 \mathrm{pg}$ per cell and $1.5 \mathrm{pg}$ per cell, and only $0.6 \mathrm{pg}$ per cell for the free boron nanoparticles). The two liposome systems therefore allow an uptake per cell that exceeds the recommended concentration of boron for effective BNCT. Finally, the toxicity of the liposomes was determined to be negligible.

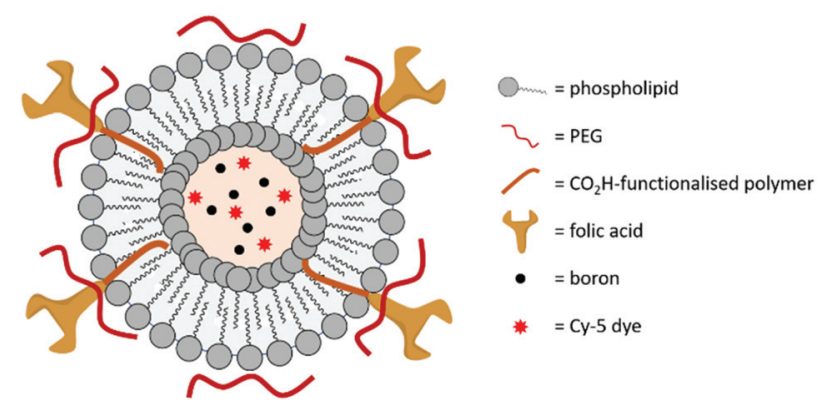

Fig. 13 Schematic representation of the boron-encapsulated liposome synthesised by Krishnan, Prasad and co-workers.

\section{Conclusions}

BNCT has not yet gained worldwide acceptance by physicians to treat cancer patients on a daily basis. There are various reasons for this. The first one is the access to neutron sources. Until recently, only nuclear reactors could deliver neutrons of the correct energy. Accelerators built on hospital sites are now suitable as neutron sources for $\mathrm{BNCT}^{12}$ and have been built in a few countries around the globe (for example, Japan and Finland). ${ }^{55}$ The second one is the lack of new and more effective boron delivery agents. BPA and BSH have been the only two compounds so far to show in vivo results promising enough in terms of biodistribution, toxicology and therapeutic effect, which then led to human studies and their clinical use. The formidable advances that have happened in the last few decades in the fields of polymer chemistry and formulation for designing drug delivery systems will undoubtedly benefit the BNCT community. Thirdly, BNCT studies have so far mainly focused on brain tumours (with an emphasis on high-grade gliomas), with more limited studies on melanomas and recurrent head and neck tumours. ${ }^{56}$ Other hard-to-treat cancers could be expected to be treated using BNCT, based on either current treatment modalities not anatomically suitable or lack of efficient treatment modalities. The author believes that from the pool of polymeric systems, boron-conjugated polymers are highly promising, especially as some polymer-drug conjugate therapeutics have already been approved. However, the path for designing a suitable system is not straightforward, especially for polymer-small drug conjugates, for which the EPR effect seems to play a vital role. Therefore, having a system loaded with enough boron is one criterion, but ensuring that this system reaches only the targeted cells in quantities high enough is another challenge. Labelling and targeting moieties will most certainly need to be taken into account in the design of successful BNCT carriers.

\section{Abbreviations}

$\begin{array}{ll}\text { ATRP } & \text { Atom transfer radical polymerisation } \\ \text { BBB } & \text { Blood-brain barrier } \\ \text { Bis-MPA } & \text { 2,2-Bis(hydroxymethyl)propanoic acid } \\ \text { BNCT } & \text { Boron neutron capture therapy } \\ \text { BPA } & \text { p-Boronophenylalanine } \\ \text { BSH } & \text { Sodium borocaptate } \\ \text { CED } & \text { Convection-enhanced delivery } \\ \text { EGFR } & \text { Epithermal growth factor receptor } \\ \text { EPR } & \text { Enhanced permeability and retention } \\ \text { FA } & \text { Folic acid } \\ \text { Gn } & \text { Generation } n \text { of a dendron } \\ \text { HVJ-E } & \text { Hemagglutinating virus of Japan Envelope } \\ \text { ICP-MS } & \text { Inductively coupled plasma-mass spectrometry } \\ \text { MST } & \text { Mean survival time } \\ \text { OEGMA } & \text { Oligo(ethylene glycol)methyl methacrylate } \\ \text { PAMAM } & \text { Poly(amidoamine) } \\ \text { PCL } & \text { Poly(caprolactone) }\end{array}$


PEG Poly(ethylene glycol)

RAFT Reversible addition-fragmentation chain-transfer

ROP Ring-opening polymerisation

VGEF Vascular growth epithermal factor

\section{Conflicts of interest}

There are no conflicts to declare.

\section{References}

1 K. Nedunchezhian, N. Aswath, M. Thiruppathy and S. Thirugnanamurthy, J. Clin. Diagn. Res., 2016, 10, ZE01ZE04.

2 K. Hu, Z. Yang, L. Zhang, L. Xie, L. Wang, H. Xu, L. Josephson, S. H. Liang and M.-R. Zhang, Coord. Chem. Rev., 2020, 405, 213139.

3 J. F. Valliant, K. J. Guenther, A. S. King, P. Morel, P. Schaffer, O. O. Sogbein and K. A. Stephenson, Coord. Chem. Rev., 2002, 232, 173-230.

4 P. G. Kruger, Proc. Natl. Acad. Sci. U. S. A., 1940, 26, 181.

5 L. E. Farr, W. H. Sweet, J. S. Robertson, C. G. Foster, H. B. Locksley, D. L. Sutherland, M. L. Mendelsohn and E. E. Stickley, Am. J. Roentgenol., 1954, 71, 279-293.

6 D. N. Slatkin, Brain, 1991, 114, 1609-1629.

7 Y. Nakagawa and H. Hatanaka, J. Neurooncol., 1997, 33, 105-115.

8 F. Alam, A. H. Soloway and R. F. Barth, Int. J. Radiat. Appl. Instrum., Part A, 1987, 38, 503-506.

9 R. F. Barth, P. Mi and W. Yang, Cancer Commun., 2018, 38, 35.

10 R. F. Barth, Z. Zhang and T. Liu, Cancer Commun., 2018, 38, 36.

11 S.-I. Miyatake, S. Kawabata, R. Hiramatsu, T. Kuroiwa, M. Suzuki, N. Kondo and K. Ono, Neurol. Med. Chir., 2016, 56, 361-371.

12 C. N. Culbertson, S. Green, A. J. Mason, D. Picton, G. Baugh, R. P. Hugtenburg, Z. Yin, M. C. Scott and J. M. Nelson, Appl. Radiat. Isot., 2004, 61, 733-738.

13 K. Hirose, A. Konno, S. Yoshimoto, K. Ono, N. Otsuki, J. Hatazawa, J. Hiratsuka and Y. Takai, Ann. Oncol., 2019, 30, v460 .

14 K. Jeet and T. R. Ronald, Curr. Org. Chem., 2010, 14, 138147.

15 X. Yang, S. J. Kalita, S. Maheshuni and Y.-Y. Huang, Coord. Chem. Rev., 2019, 392, 35-48.

16 F. Cheng and F. Jäkle, Polym. Chem., 2011, 2, 2122-2132.

17 R. Núñez, I. Romero, F. Teixidor and C. Viñas, Chem. Soc. Rev., 2016, 45, 5147-5173.

18 M. C. Parrott, E. B. Marchington, J. F. Valliant and A. Adronov, Macromol. Symp., 2003, 196, 201-211.

19 S. E. A. Gratton, M. C. Parrott and A. Adronov, J. Inorg. Organomet. Polym. Mater., 2005, 15, 469-475.
20 H. Xiong, X. Wei, D. Zhou, Y. Qi, Z. Xie, X. Chen, X. Jing and Y. Huang, Bioconjugate Chem., 2016, 27, 2214-2223.

21 D. E. Morrison, F. Issa, M. Bhadbhade, L. Groebler, P. K. Witting, M. Kassiou, P. J. Rutledge and L. M. Rendina, J. Biol. Inorg. Chem., 2010, 15, 1305-1318.

22 Z. Ruan, L. Liu, L. Fu, T. Xing and L. Yan, Polym. Chem., 2016, 7, 4411-4418.

23 G. Chen, J. Yang, G. Lu, P. C. Liu, Q. Chen, Z. Xie and C. Wu, Mol. Pharm., 2014, 11, 3291-3299.

24 S. Yoneoka, Y. Nakagawa, K. Uto, K. Sakura, T. Tsukahara and M. Ebara, Sci. Technol. Adv. Mater., 2019, 20, 291-304.

25 Y. Kaneda, T. Nakajima, T. Nishikawa, S. Yamamoto, H. Ikegami, N. Suzuki, H. Nakamura, R. Morishita and H. Kotani, Mol. Ther., 2002, 6, 219-226.

26 L.-C. S. Huang, D. Le, I.-L. Hsiao, S. Fritsch-Decker, C. Hald, S.-C. Huang, J.-K. Chen, J. R. Hwu, C. Weiss, M.-H. Hsu and G. Delaittre, Polym. Chem., 2021, 12, $50-56$.

27 F. Alam, A. H. Soloway, R. F. Barth, N. Mafune, D. M. Adams and W. H. Knoth, J. Med. Chem., 1989, 32, 2326-2330.

28 S. Novick, M. R. Quastel, S. Marcus, D. Chipman, G. Shani, R. F. Barth and A. H. Soloway, Nucl. Med. Biol., 2002, 29, 159-167.

29 D. J. Worm, P. Hoppenz, S. Els-Heindl, M. Kellert, R. Kuhnert, S. Saretz, J. Köbberling, B. Riedl, E. HeyHawkins and A. G. Beck-Sickinger, J. Med. Chem., 2020, 63, 2358-2371.

30 D. Astruc, E. Boisselier and C. Ornelas, Chem. Rev., 2010, 110, 1857-1959.

31 G. Wu, R. F. Barth, W. Yang, M. Chatterjee, W. Tjarks, M. J. Ciesielski and R. A. Fenstermaker, Bioconjugate Chem., 2004, 15, 185-194.

32 F. Alam, A. H. Soloway, J. E. McGuire, R. F. Barth, W. E. Carey and D. Adams, J. Med. Chem., 1985, 28, 522525.

33 J. Capala, R. F. Barth, M. Bendayan, M. Lauzon, D. M. Adams, A. H. Soloway, R. A. Fenstermaker and J. Carlsson, Bioconjugate Chem., 1996, 7, 7-15.

34 R. F. Barth, W. Yang, D. M. Adams, J. H. Rotaru, S. Shukla, M. Sekido, W. Tjarks, R. A. Fenstermaker, M. Ciesielski, M. M. Nawrocky and J. A. Coderre, Cancer Res., 2002, 62, 3159-3166.

35 W. Yang, R. F. Barth, D. M. Adams, M. J. Ciesielski, R. A. Fenstermaker, S. Shukla, W. Tjarks and M. A. Caligiuri, Cancer Res., 2002, 62, 6552-6558.

36 R. F. Barth, D. M. Adams, A. H. Soloway, F. Alam and M. V. Darby, Bioconjugate Chem., 1994, 5, 58-66.

37 Y. Dai, C. Xu, X. Sun and X. Chen, Chem. Soc. Rev., 2017, 46, 3830-3852.

38 M. V. Backer, T. I. Gaynutdinov, V. Patel, A. K. Bandyopadhyaya, B. T. S. Thirumamagal, W. Tjarks, R. F. Barth, K. Claffey and J. M. Backer, Mol. Cancer Ther., 2005, 4, 1423.

39 S. Tran, P.-J. DeGiovanni, B. Piel and P. Rai, Clin. Transl. Med., 2017, 6, 44. 
40 S. Shukla, G. Wu, M. Chatterjee, W. Yang, M. Sekido, L. A. Diop, R. Müller, J. J. Sudimack, R. J. Lee, R. F. Barth and W. Tjarks, Bioconjugate Chem., 2003, 14, 158-167.

41 B. P. Dash, R. Satapathy, B. P. Bode, C. T. Reidl, J. W. Sawicki, A. J. Mason, J. A. Maguire and N. S. Hosmane, Organometallics, 2012, 31, 2931-2935.

42 N. Li, P. Zhao, L. Salmon, J. Ruiz, M. Zabawa, N. S. Hosmane and D. Astruc, Inorg. Chem., 2013, 52, 11146-11155.

43 M. C. Parrott, E. B. Marchington, J. F. Valliant and A. Adronov, J. Am. Chem. Soc., 2005, 127, 12081-12089.

44 Y. Ma, Q. Mou, D. Wang, X. Zhu and D. Yan, Theranostics, 2016, 6, 930-947.

45 L. Ma, J. Hamdi, F. Wong and M. F. Hawthorne, Inorg. Chem., 2006, 45, 278-285.

46 S. R. Benhabbour and A. Adronov, Polymers for Biomedical Applications, American Chemical Society, 2008, vol. 977, ch. 14, pp. 238-249.

47 L. M. Bronstein and Z. B. Shifrina, Chem. Rev., 2011, 111, 5301-5344.
48 T. Sun, Y. Li, Y. Huang, Z. Zhang, W. Yang, Z. Du and Y. Zhou, Oncotarget, 2016, 7, 43095-43108.

49 H. Fujii, A. Matsuyama, H. Komoda, M. Sasai, M. Suzuki, T. Asano, Y. Doki, M. Kirihata, K. Ono, Y. Tabata, Y. Kaneda, Y. Sawa and C. M. Lee, Radiat. Oncol., 2011, 6, 8. 50 D. Bobo, K. J. Robinson, J. Islam, K. J. Thurecht and S. R. Corrie, Pharm. Res., 2016, 33, 2373-2387.

51 H. Yanagië, T. Tomita, H. Kobayashi, Y. Fujii, T. Takahashi, K. Hasumi, H. Nariuchi and M. Sekiguchi, Br. J. Cancer, 1991, 63, 522-526.

52 K. Shelly, D. A. Feakes, M. F. Hawthorne, P. G. Schmidt, T. A. Krisch and W. F. Bauer, Proc. Natl. Acad. Sci. U. S. A., 1992, 89, 9039.

53 H. Nakamura, Future Med. Chem., 2013, 5, 715-730.

54 A. Singh, B. K. Kim, Y. Mackeyev, P. Rohani, S. D. Mahajan, M. T. Swihart, S. Krishnan and P. N. Prasad, J. Biomed. Nanotechnol., 2019, 15, 1714-1723.

55 M. Suzuki, Int. J. Clin. Oncol., 2020, 25, 43-50.

56 R. F. Barth and J. C. Grecula, Appl. Radiat. Isot., 2020, 160, 109029. 